\title{
The TAS-20 more likely measures negative affects rather than alexithymia itself in patients with major depression, panic disorder, eating disorders and substance use disorders
}

\author{
Carlo Marchesi ${ }^{\mathrm{a}, \mathrm{b}, *}$, Paolo Ossola ${ }^{\mathrm{a}}$, Matteo Tonna ${ }^{\mathrm{b}}$, Chiara De Panfilis ${ }^{\mathrm{a}, \mathrm{b}}$ \\ ${ }^{a}$ Department of Neuroscience, Psychiatry Unit, University of Parma, Parma, Italy \\ ${ }^{\mathrm{b}}$ Mental Health Department, AUSL, Parma, Italy
}

\begin{abstract}
Background: This study evaluates whether the difference in Toronto Alexithymia Scale-20 item (TAS-20) between patients with major depression (MD), panic disorder (PD), eating disorders (ED), and substance use disorders (SUD) and healthy controls persisted after controlling for the severity of anxiety and depression.

Methods: Thirty-eight patients with MD, 58 with PD, 52 with ED, and 30 with SUD and 78 healthy controls (C) completed the TAS-20, the Hamilton Rating Scale for Anxiety (Ham-A), the Hamilton Rating Scale for Depression (Ham-D).

Results: The differences in TAS-20 scores observed between patient groups, regardless of the type of their disorders, and controls disappeared after controlling for the effect of anxiety and depression severity. In contrast, the differences in severity of anxiety and depression between patients and controls were still present, after excluding the effect of alexithymic levels.

Conclusions: Our data suggest that alexithymic levels, as measured by the TAS-20, are modulated by the severity of symptoms, supporting the view that alexithymia can represent a state phenomenon in patients with MD, PD, ED and SUD, because the TAS-20 seems overly sensitive to a general distress syndrome, and it is more likely to measure negative affects rather than alexithymia itself.
\end{abstract}

(C) 2014 Elsevier Inc. All rights reserved.

\section{Introduction}

Alexithymia is a multidimensional construct characterized by an impoverished fantasy life, difficulty in expressing or naming feelings, difficulty distinguishing between bodily sensations and feelings, and a preoccupation with external events [1]. The TAS-20 is the most widely used and studied self-report measure of alexithymia. Factor analysis has supported three-factor solutions for this scale: the factors represent (1) difficulty identifying feelings (DIF), (2) difficulty communicating and describing feelings (DDF), and (3) external-oriented thinking (EOT). Items representing impo-

Conflict of interests: No authors received funding for the research and had financial involvement that could represent potential conflicts of interest.

* Corresponding author at: Department of Neuroscience, Psychiatry Unit, General Hospital, Università di Parma, Braga Building, Via Gramsci 14, 43100 Parma, Italy. Tel.: +39 0521903597 98; fax: +39 0521396825.

E-mail address: carlo.marchesi@unipr.it (C. Marchesi). verished fantasy or reduced daydreaming were dropped from the TAS-20 based on the factor analyses solutions.

Alexithymia, reflecting a disordered affect regulation, is thought to increase vulnerability to psychological illness, especially in conflict-afflicted psychosocial situations. Therefore, people with alexithymic personality are supposed to be at risk for developing mental disorders such as major depression [1], panic disorder [2], eating disorders [3] and substance use disorders [4].

Concerning major depression (MD) the rate of alexithymia ranges between $45 \%$ and $46 \%$ during the acute phase of illness [5-11]. A similar rate of alexithymia (29\%-44\%) was found in the active phase of panic disorder (PD) $[2,12]$, and even higher rates $(23 \%-77 \%)$ were observed in patients with eating disorders (ED) (anorexia nervosa, bulimia nervosa and binge eating disorder) [13-16] and in patients with substance use disorders (SUD) (i.e., between $45 \%$ and $67 \%$ of patients with alcohol use disorders) [17].

However, the evaluation of alexithymia with the TAS-20 can be limited by the fact that alexithymia cannot be validly 
assessed by a self-report instrument because people with alexithymia, by definition, should not be able to report their psychological state.

Further, when alexithymia was assessed with the TAS-20, significant relationships have been found with depression and anxiety not only in the general population $[18,19]$, but also in clinical samples. In the general population, depressive symptoms explained almost $36 \%$ of the variance in alexithymic features [18] or were significantly correlated with all alexithymic dimensions (DIF: $r=0.52$; DDF: $r=$ 0.42; EOT: $r=0.20$ ) [19].

In clinical samples, some studies observed a decrease in the TAS-20 scores when depressive symptoms improved in MD [5-11,20] or when anxiety symptoms decreased in PD [2]. Moreover, an overlap between TAS-20 (particularly the DIF dimension) and anxiety was found in a factor analysis study in patients with anxiety or depressive disorders [25], and an association between alexithymia and severity of depression and anxiety was also found in SUD [21-24] and in $\operatorname{ED}[3,14]$.

Altogether the aforementioned findings raise the question whether the TAS-20 measures negative affects rather than alexithymia itself in mental disorder. Hoffart [26] and Lumley [27] suggest a method to answer this question: if the TAS-20 identifies alexithymic traits the differences between patients and healthy controls should persist after controlling for anxiety and depression. To our knowledge, however, no study investigating alexithymia in mental disorders with the TAS-20 applied this method.

Therefore the present study aimed to clarify whether the differences in TAS-20 scores among patients with MD, PD, ED and SUD and healthy controls remained after controlling for the effect of the severity of anxious and depressive symptoms.

\section{Materials and methods}

\subsection{Sample}

Patients were recruited from all patients who consecutively sought treatment at the outpatient service of the Psychiatry Clinic of the University of Parma-Italy from January to December 2011, after receiving a diagnosis of $\mathrm{MD}$, PD, ED or SUD. The local ethics committee approved the study protocol and the study was conducted according to the Helsinki Declaration. All patients provided informed consent.

Exclusion criteria for study participation were the presence of schizophrenia or other psychotic disorders, organic mental disorders, severe suicidal risk, a history of neurological or medical disorder, a BMI lower than 16 for ED patients and abstinence from substances for less than 2 months for SUD subjects.

The control group comprised age- and sex-matched healthy subjects, recruited from university and hospital employers.

\subsection{Assessment}

After giving informed consent, all subjects were administered the Structured Clinical Interview for DSM-IV Disorders [28], the Hamilton Rating Scale for Anxiety (Ham-A) [29] and for Depression (Ham-D) [30], the 20 item-Toronto Alexithymia Scale (TAS-20) [31] and a semi-structured interview assessing clinical and anamnestic information.

A resident in psychiatry (P.O.) was specifically trained to administer the SCID. The training consisted in the administration of the SCID to 10 patients affected by major depression, dysthymia, panic disorder, generalized anxiety disorder, anorexia nervosa, bulimia nervosa, binge eating disorder, cocaine or heroin dependence. The interviews were audiotaped and reviewed by a senior psychiatrist (C.M.). A satisfactory diagnostic reliability $(k=0.79)$ was obtained during the training.

\subsection{Statistical analysis}

The internal consistency for the TAS-20 was calculated in each diagnostic group. The Cronbach $\alpha$ was .78 in the MD group, .87 in the PD group, .83 in the ED group, .81 in the UD group and .80 in the control group. The values obtained in each group demonstrated that patients were able to understand the questions and to respond to the items in an appropriate and consistent way. Differences among the diagnostic groups were evaluated using $\chi^{2}$ for categorical variables and one-way ANOVA with Bonferroni post hoc analysis for continuous variables, as appropriate.

We used Pearson correlation to evaluate how alexithymia was related to age and education.

We used analysis of covariance (ANCOVA) to evaluate the between-groups differences on TAS-20 scores after adjusting for age and education, which were found to be significantly different among diagnostic groups. Moreover, we then used analysis of covariance (ANCOVA) to evaluate the betweengroups differences on the following: (1) TAS-20 scores, after controlling for age, gender, education and severity of anxious and depressive symptoms (Ham-A and Ham-D scores), and (2) Ham-A and Ham-D scores, after controlling for the effect TAS-20 total, DIF, DDF, and EOT scores.

Finally, linear regression analysis was used to evaluate the relationship between TAS-20 total, DIF, DDF and EOT scores (dependent variables) and age, gender $(0=$ male; $1=$ female), years of education, Ham-A and Ham-D scores (independent variables) in the whole sample, in order to establish whether alexithymia levels were associated with the severity of concurrent anxious and depressive symptoms.

\section{Results}

\subsection{Sample}

A total of 178 patients and 78 healthy controls (C) participated in this study. Within the clinical group, 38 patients were affected by MD, 58 by PD, 52 by ED (anorexia 
nervosa $n=20$; bulimia nervosa $n=20$; binge eating disorder $n=14$ ) and 30 by SUD (cocaine dependence $n=$ 10 ; heroin dependence $n=11$; multiple substances dependence $n=9$ ). Table 1 shows the socio-demographic features of both the patient and the control groups.

\subsection{TAS-20 scores}

TAS-20 total score was higher in MD, PD and ED patients than in C, while the difference between SUD and C did not reach statistical significance (Table 2). Patients with MD showed the highest levels in all three subscales of TAS-20, whereas ED reported higher scores than C only for DIF and DDF, PD only for DIF dimension, and SUD only for DDF dimension. All these differences persisted after controlling for age and education (ANCOVA, TAS-20 total score: $F=16.8$; $p<.001$; DIF: $F=22.4 ; p<.001$; DDF: $F=7.6 ; p<.001$; EOT: $F=5.5 ; p<.001$ ), which significantly differed among diagnostic groups, although only age correlated with the TAS20 scores $(b=.14 ; p=.02)$. Among groups, all the abovementioned differences in TAS-20 scores disappeared after controlling for the effect of severity of anxious and depressive symptoms (Table 2).

\subsection{Severity of anxious symptoms}

Ham-A scores were significantly higher in patients than in C. Among patients, the most anxious were those with MD and the lowest those with SUD, with PD and ED lying in the middle position (Table 2). The significant differences among groups were still present after controlling for the effect of age, gender, years of education DIF, DDF, and EOT scores (Table 2).

\subsection{Severity of depressive symptoms}

The Ham-D scores were significantly higher in MD patients than in PD, ED, SUD patients or in C. Moreover, PD, ED, SD patients were more depressed than C (Table 2).
The significant differences among groups were still present after controlling for the effect of age, gender, years of education, DIF DDF, and EOT TAS-20 scores (Table 2).

\subsection{Relationship between TAS-20 scores and anxiety or depression}

In the total sample, linear regression analyses showed that TAS-20 scores were predicted by Ham-A scores $(\beta=$ $.28 ; t=3.0 ; p=.002)$ and Ham-D scores $(\beta=.23 ; t=2.5$; $p=.01)$; DIF scores were predicted by Ham-A scores $(\beta=.47 ; t=5.3 ; p<.001)$ and by female gender $(\beta=.15$; $t=2.7 ; p=.006)$; DDF and EOT scores were both predicted by Ham-D scores $(\beta=.23 ; t=2.2 ; p=.02$; and $\beta=.21$; $t=2.1 ; p=.03$, respectively) and age $(\beta=.17 ; t=2.7$; $p=.007)$.

\section{Discussion}

This study evaluated whether patients with psychiatric disorders (MD, PD, ED, SUD) show higher TAS-20 scores than healthy controls even when controlling for the potential confounding effect of anxious and depressive symptoms.

The results indicate that all patient groups, regardless of their specific disorder, reported higher TAS-20 scores than healthy $\mathrm{C}$, although the difference between SUD patients and $\mathrm{C}$ did not reach a statistical significance.

This finding confirms the results of previous studies that assessed alexithymia with the TAS-20 in MD [5-11,32], PD $[2,12]$, ED [13-16] and SUD [17,23,24]. However, the differences on TAS-20 total score observed among the diagnostic groups disappeared after controlling for the effect of anxious and depressive symptoms. This finding suggests that the severity of anxious and depressive symptomatology could account for the increased TAS-20 scores observed in patients with different mental disorders: when the differences in symptoms severity were controlled for, all patient groups,

Table 1

Socio-demographic and psychopathological characteristics of patients with major depression (MD), panic disorder (PD), eating disorder (ED), and substance dependence (SD) and healthy controls (C).

\begin{tabular}{|c|c|c|c|c|c|c|}
\hline & $\operatorname{MD}(n=38)$ & $\mathrm{PD}(n=58)$ & $\mathrm{ED}(n=52)$ & $\mathrm{SD}(n=30)$ & $\mathrm{C}(n=78)$ & \\
\hline Gender (female), $n(\%)$ & $19(50.0)$ & $40(69.0)$ & $48(92.3)$ & $6(20.0)$ & $63(80.8)$ & $\chi^{2}=58.0 ; p<.001$ \\
\hline Age (years) & $51.3 \pm 11.7$ & $36.3 \pm 10.7$ & $43.5 \pm 12.5$ & $33.2 \pm 9.6$ & $41.2 \pm 11.8$ & $F=14.0 ; p<.001$ \\
\hline Education (years) & $9.9 \pm 4.4$ & $10.3 \pm 3.5$ & $10.3 \pm 3.5$ & $8.7 \pm 3.0$ & $8.4 \pm 3.6$ & $F=3.6 ; p=.007$ \\
\hline \multicolumn{6}{|l|}{ Family status, $n(\%)$} & $\chi^{2}=58.5 ; p<.001$ \\
\hline Never married & $9(23.7)$ & $19(32.8)$ & $12(23.1)$ & $27(90.0)$ & $22(28.2)$ & \\
\hline Married & $20(52.6)$ & $30(51.7)$ & $34(65.4)$ & $2(6.7)$ & $48(61.5)$ & \\
\hline Separated/divorced & $8(21.1)$ & $7(12.1)$ & $4(7.7)$ & $1(3.3)$ & $3(3.8)$ & \\
\hline Widowed & $1(2.6)$ & $2(3.4)$ & $2(3.8)$ & - & $5(6.4)$ & \\
\hline \multicolumn{6}{|l|}{ Occupation, $n(\%)$} & $\chi^{2}=64.1 ; p<.001$ \\
\hline Unemployed & $5(13.2)$ & $5(8.6)$ & $4(7.7)$ & $13(43.3)$ & $5(6.4)$ & \\
\hline Student & - & $9(15.5)$ & $3(5.8)$ & $1(3.3)$ & $7(9.0)$ & \\
\hline Housewife & 7 (18.4) & $6(10.3)$ & $3(5.8)$ & $1(3.3)$ & $18(23.1)$ & \\
\hline Employed & $19(50.0)$ & $38(65.5)$ & $38(73.1)$ & $15(50.0)$ & $43(55.1)$ & \\
\hline Retired & $7(18.4)$ & - & $4(7.7)$ & - & $5(6.4)$ & \\
\hline \multicolumn{7}{|l|}{ Alexithymia, $n(\%)$} \\
\hline TAS-20 score $>60$ & $17(44.7)$ & $16(27.6)$ & $17(32.7)$ & $5(16.7)$ & $4(5.1)$ & $\chi^{2}=28.2 ; p<.001$ \\
\hline
\end{tabular}


Table 2

Severity of depressive-anxious symptoms and levels of alexithymia in patients with major depression (MD, panic disorder (PD), eating disorders (ED), substance use disorders (SUD and in healthy controls (C).

\begin{tabular}{cllllll}
\hline & MD $(n=38)$ & PD $(n=58)$ & ED $(n=52)$ & SUD $(n=30)$ & C $(n=78)$ & One-way ANOVA $(d f=4,251)$ \\
\hline Ham-A & $25.1 \pm 8.1$ & $20.1 \pm 7.0$ & $16.5 \pm 4.7$ & $11.3 \pm 5.1$ & $1.9 \pm 2.3$ & $F=153.9 ; p<.001^{\mathrm{a}}$ \\
Ham-D & $24.7 \pm 6.6$ & $13.7 \pm 6.1$ & $11.5 \pm 4.7$ & $13.1 \pm 5.2$ & $1.9 \pm 2.0$ & $F=147.7 ; p<.001^{\mathrm{b}}$ \\
TAS-20 & & & & & \\
DIF & $21.5 \pm 7.2$ & $20.9 \pm 6.4$ & $20.2 \pm 6.7$ & $16.1 \pm 6.2$ & $13.0 \pm 4.8$ & $F=21.6 ; p<.001^{\mathrm{c}}$ \\
DDF & $16.0 \pm 4.8$ & $12.6 \pm 4.4$ & $13.7 \pm 5.5$ & $14.3 \pm 4.0$ & $11.0 \pm 4.6$ & $F=7.9 ; p<.001^{\mathrm{d}}$ \\
EOT & $21.3 \pm 3.0$ & $18.7 \pm 6.3$ & $18.7 \pm 6.3$ & $17.7 \pm 4.4$ & $16.0 \pm 5.9$ & $F=5.9 ; p<.001^{\mathrm{e}}$ \\
Total & $58.9 \pm 12.1$ & $52.2 \pm 14.4$ & $53.0 \pm 14.4$ & $47.4 \pm 11.4$ & $40.1 \pm 11.5$ & $F=16.9 ; p<.001^{\mathrm{f}}$
\end{tabular}

Means adjusted for the effects of covariates

\begin{tabular}{|c|c|c|c|c|c|c|}
\hline \multicolumn{6}{|c|}{ Means adj } & \multirow{2}{*}{$\begin{array}{l}\text { ANCOVA } \\
(d f=10,256)\end{array}$} \\
\hline \multirow[b]{2}{*}{ Ham-A } & \multicolumn{5}{|c|}{ Covariates: age, gender, education, DIF, DCF and EOT scores } & \\
\hline & $24.7 \pm 5.5$ & $19.2 \pm 5.3$ & $15.7 \pm 5.0$ & $11.9 \pm 5.4$ & $3.0 \pm 5.2$ & $F=88.4 ; p<.001^{\mathrm{a}}$ \\
\hline \multirow[t]{2}{*}{ Ham-D } & $23.8 \pm 4.9$ & $13.4 \pm 4.5$ & $11.0 \pm 4.3$ & $13.9 \pm 4.9$ & $2.6 \pm 5.2$ & $F=90.6 ; p<.001^{\mathrm{b}}$ \\
\hline & \multicolumn{5}{|c|}{ Covariates: age, gender, education, Ham-A and Ham-D scores } & $\operatorname{ANCOVA}(d f=9,256)$ \\
\hline \multicolumn{7}{|l|}{ TAS-20 } \\
\hline DIF & $17.8 \pm 9.2$ & $19.2 \pm 6.0$ & $19.2 \pm 5.7$ & $17.1 \pm 6.5$ & $16.3 \pm 9.7$ & $F=1.4 ; p=.21$ \\
\hline DDF & $14.9 \pm 7.3$ & $12.0 \pm 5.3$ & $13.5 \pm 5.0$ & $14.1 \pm 5.4$ & $12.1 \pm 7.9$ & $F=1.9 ; p=.10$ \\
\hline EOT & $18.5 \pm 8.6$ & $19.0 \pm 6.9$ & $19.0 \pm 5.7$ & $17.1 \pm 6.0$ & $17.3 \pm 8.8$ & $F=0.6 ; p=.60$ \\
\hline Total & $51.3 \pm 19.7$ & $50.2 \pm 14.4$ & $52.1 \pm 12.9$ & $47.8 \pm 14.2$ & $45.7 \pm 21.1$ & $F=1.0 ; p=.38$ \\
\hline
\end{tabular}

DIF, difficulty identifying feelings; DDF, difficulty communicating and describing feelings; EOT, external oriented thinking.

Bonferroni post hoc analysis.

${ }^{\mathrm{a}} \mathrm{D}>\mathrm{PD}>\mathrm{ED}>\mathrm{SD}>\mathrm{C}$.

${ }^{\mathrm{b}} \mathrm{D}>\mathrm{PD}, \mathrm{ED}, \mathrm{SD}>\mathrm{C}$.

${ }^{c} \mathrm{D}, \mathrm{PD}, \mathrm{ED}>\mathrm{SD}, \mathrm{C}$.

${ }^{\mathrm{d}} \mathrm{D}>\mathrm{PD}, \mathrm{C} ; \mathrm{ED}, \mathrm{SD}>\mathrm{C}$.

${ }^{\mathrm{e}} \mathrm{D}>\mathrm{C}$.

${ }^{\mathrm{f}} \mathrm{D}>\mathrm{SD}, \mathrm{C} ; \mathrm{PD}, \mathrm{ED}>\mathrm{C}$.

regardless of their disorder, showed TAS-20 total scores comparable to those reported by healthy controls.

At the same time, our results do not support the notion that TAS-20 scores increase the risk for anxiety and depression as suggested by some authors [33,34]. In order to test this hypothesis, we also evaluated whether the observed difference between patients and controls in anxious and depressive psychopathology disappeared after controlling for the effect of TAS-20 scores. Results showed that this was not the case in our sample, suggesting that TAS-20 levels do not influence the severity of anxious and depressive symptoms.

Furthermore, the patient groups scored differently on the three alexithymic dimensions.

Specifically, MD patients reported higher DIF, DDF and EOT than controls, whereas ED patients showed an increase only in DIF and DDF. PD patients were higher than controls only on DIF, and SUD only on DDF. However, all these observed differences among groups disappeared after excluding the effect of symptoms severity, suggesting that psychopathology severity was responsible for the increased levels of the alexithymic dimensions. Thus, the different patterns of alexithymia reported by diverse diagnostic groups could be explained by the different effects of anxious and depressive symptoms on the three alexithymic dimensions.
In fact, in this study the severity of anxiety directly predicted DIF scores $(\beta=.47 ; p<.001)$, whereas the severity of depression predicted DDF $(\beta=.21 ; p=.03)$ and EOT scores. Accordingly, the highest DIF, DDF, EOT scores were found in MD patients, who also reported the highest severity of anxious and depressive symptoms, while PD patients, who were more anxious than depressed, were higher than controls only on the DIF dimension, and SUD patients, who were more depressed than anxious, were higher than controls only with respect to DDF scores. Taken together, these findings support the hypothesis of a specific relationship between some anxiety and depressive symptoms and definite alexithymic dimensions. Indeed, previous studies $[23,35,36]$ reported an association between psychic anxiety and DIF dimension. Further, DDF elevation has been associated with suicide ideation in depressed patients [37] as well as with depersonalization/derealization in PD patients [30] whereas the EOT dimension has been positively related to psychomotor retardation in major depressed patients [38].

The results of this study have the potential to contribute to the ongoing and long-lasting debate concerning the stability of alexithymia among mental disorders. Several influential authors $[1,9,11,39]$ suggest that alexithymia is a personality trait that, as such, is characterized by a relative stability: while alexithymic levels can increase and decrease depending on 
the fluctuation of illness symptoms, the relative differences among individuals remain stable over time. This hypothesis implies that the basic personality conditions, which constitute a liability toward a mental disorder, are accentuated by the state of illness and return to the pre-existing conditions after remission, as suggested by Hoffart [26]. According to this trait-state hypothesis [26] a basic personality condition constitutes a predispositional factor to the disorder whether "the observed personality differences between diagnostic groups will persist after symptom severity has been controlled for." With respect to alexithymia, this means that if alexithymia was a personality trait in a mental disorder the difference in TAS-20 scores observed between patients and healthy controls should still hold true after excluding the effect of illness symptoms. For instance, Lumley [27] suggested to test the uniqueness of the TAS in the relationship with depression by statistically controlling the other construct and examining the residual relationship of the TAS with the criterion. In the present study, the differences in TAS-20 scores among patients and between patients and healthy controls disappeared, after controlling for the effect of the severity of anxious and depressive symptoms. Therefore, our findings do not support the hypothesis that alexithymia behaves as a stable personality trait among patients with psychiatric disorders; on the contrary, these data indicate that alexithymia, as assessed by the TAS-20, is a state phenomenon, because its levels appear to be modulated by the severity of symptoms (and not vice versa). This confirms our previous findings that among women who developed MD [20] or PD [40] alexithymia levels were in the normal range prior to illness onset, but increased steadily during the acute phase of the disorder.

There are at least two alternative explanations for the observed relationship between alexithymia and anxiety and depression. Alexithymia may be a temporary response to stress represented by an illness episode; in this view "secondary alexithymia" can represent a defense or a strategy to cope with distress (emotional pain, aversive memories and physiological arousal) associated with a mental disorder [25]. In the second view, the relationship between alexithymia and depression may represent an artifact of the method and measures used [27], since, particularly, the TAS-20 dimensions DIF and DDF are associated with different measures of negative affects $[2,3,14,16,18,20,25,27,39,41-45]$. Therefore individuals with negative emotional states (i.e. anxiety and depression) might score high on these TAS-20 dimensions.

The present study supports this latter view, since also in mental disorders such as ED and SUD, which are frequently associated with anxiety and depression, the higher levels of TAS-20 scores disappeared when the effect of anxiety and depression was excluded. This should have not occurred if alexithymia was a personality trait predisposing people to ED or SUD, as hypothesized by previous studies [46-48].

Thus, our study confirms the shortcomings with respect to validity and reliability of TAS-20 in the assessment of alexithymia in clinical samples [49,50]: alexithymia, as assessed by the TAS-20, can be more a measure of negative affect rather than a measure of deficit in the cognitive processing of emotions across different mental disorders. This seems particularly true for the DIF and DDF dimensions, since DIF capture anxiety symptoms and DDF is sensible to depressive symptoms. According to this view, the proposed existence of an alexithymic depression [51], characterized by high DIF and DDF levels, may be questioned, since in the study of Vanheule et al. [51], even though the alexithymic patients showed the most severe depressive symptoms, the TAS-20 differences between strongly and moderately alexithymic patients were not adjusted the for the severity of anxious and depressive symptoms. Similarly, also the conclusion of a recent study can be questioned [52]. In the study of Leweke et al. [52], the increased prevalence of alexithymic subjects in mental disorders, especially in depressive disorders, let the authors to conclude that alexithymia may be associated with a higher vulnerability to mental illness, even though they did not control if the high prevalence of alexithimic subject persisted in mental disorders after adjusting for the severity of anxious and depressive symptoms. Therefore, no conclusion about the causal relationship between alexithymia and mental disorders can be drawn from these two studies [51,52], since the effect of negative affects on the TAS-20 scores was not controlled for.

\section{Conclusion}

The present study supports the hypothesis that the TAS20 , the most widely used self-administered scale assessing alexithymia, is overly sensitive to a general distress syndrome, and therefore it is more likely to measure negative affects (distress, nervousness, fear, anger, guilt, sadness, scornfulness) rather than alexithymia itself. Future studies investigating alexithymia among psychiatric populations should control for the severity of anxiety and depression before arguing that alexithymia is a personality trait predisposing to mental disorders. Finally, the findings of the present study, based only on a self-reported measure of alexithymia, need to be confirmed using observer ratings (e.g., Toronto Structured Interview for Alexithymia) or objective performance-based tasks such as the Levels of Emotional Awareness Scale.

\section{References}

[1] Taylor GJ, Bagby RM. New trends in alexithymia research. Psychother Psychosom 2004;73:68-77.

[2] Marchesi C, Fontò S, Balista C, Cimmino C, Maggini C. Relationship between alexithymia and panic disorder: a longitudinal study to answer an open question. Psychother Psychosom 2005;74:56-60.

[3] Bydlowski S, Corcos M, Jeammet P, Paterniti S, Berthoz S, Laurier C, et al. Emotion-processing deficits in eating disorders. Int $\mathrm{J}$ Eat Disord 2005;37:321-9. 
[4] Speranza M, Corcos M, Stephan P, Loas G, Perez-Diaz F, Lang F, et al. Alexithymia, depressive experiences, and dependency in addictive disorders. Subst Use Misuse 2004;39:551-79.

[5] Honkalampi K, Hintikka J, Saarinen P, Lehtonen J, Viinämaki H. Is alexithymia a permanent feature in depressed patients? Psychother Psychosom 2000;69:303-8.

[6] Saarijärvi S, Salminen JK, Toikka TB. Alexithymia and depression. A 1-year follow-up study in outpatients with major depression. J Psychosom Res 2001;51:729-33.

[7] Honkalampi K, Hintikka J, Antikainen R, Lehtonen J, Viinämaki H. Alexithymia in patients with major depressive disorder and comorbid cluster C personality disorders: a 6-month follow-up study. J Pers Disord 2001;15:245-54.

[8] Honkalampi K, Hintikka J, Laukkanen E, Lehtonen J, Viinämaki H. Alexithymia and depression. A perspective study of patients with major depressive disorder. Psychosomatics 2001;42:229-34.

[9] Luminet O, Bagby RM, Taylor GJ. An evaluation of the absolute and relative stability of alexithymia in patients with major depression. Psychother Psychosom 2001;70:254-60.

[10] Honkalampi K, Koivumaa-Honkanen H, Antikainen R, Haatainen K, Hintikka J, Viinamaki H. Relationships among alexithymia, adverse childhood experiences, sociodemographic variables, and actual mood disorder: a 2-year clinical follow-up study of patients with major depressive disorder. Psychosomatics 2004;45:197-204.

[11] Saarijärvi S, Salminen JK, Toikka TB. Temporal stability of alexithymia over a five-year period in outpatients with major depression. Psychother Psychosom 2006;75:107-12.

[12] Galderisi S, Mancuso F, Mucci A, Garramone S, Zamboli R, Maj M. Alexithymia and cognitive dysfunctions in patients with panic disorder. Psychother Psychosom 2008;77:182-8.

[13] Pinaquy S, Chabrol H, Simon C, Louvet JP, Barbe P. Emotional eating, alexithymia and binge eating disorder in obese women. Obes Res 2003;11:195-201.

[14] Eizaguirre AE, De Cabezon AO, De Alda IO, Olariaga LJ, Maite J. Alexithymia and its relationships with anxiety and depression in eating disorder. Pers Indiv Differ 2004;36:321-31.

[15] Carano A, De Berardis D, Gambi F, Di Paolo C, Campanella D, Pelusi L, et al. Alexithymia and body image in adult outpatients with binge eating disorder. Int J Eat Disord 2006;39:332-40.

[16] Parling T, Mortazavi M, Ghaderi A. Alexithymia and emotional awareness in anorexia nervosa: time for a shift in the measurement of the concept? Eat Behav 2010;11:205-10.

[17] Thorberg FA, Young RM, Sullivan KA, Lyvers M. Alexithymia and alcohol use disorders: a critical review. Addict Behav 2009;34: 237-45.

[18] Honkalampi K, Koivumaa-Honkanen H, Lehto SM, Hintikka J, Haatainen K, Rissanen T, et al. Is alexithymia a risk factor for major depression, personality disorder, or alcohol use disorders? A prospective population-based study. J Psychosom Res 2010;68: 269-73.

[19] Deno M, Miyashita M, Fujisawa D, Nakajima S, Ito M. The relationships between complicated grief, depression, and alexithymia according to the seriousness of complicated grief in the Japanese general population. J Affect Disord 2011;135:122-7.

[20] Marchesi C, Bertoni S, Maggini C. Major and minor depression in pregnancy. Obstet Gynecol 2009;113:1292-8.

[21] Haviland MG, MacMurray JP, Cummings MA. The relationship between alexithymia and depressive symptoms in a sample of newly abstinent alcoholic inpatients. Psychother Psychosom 1988; 49:37-40.

[22] de Timary P, Luts A, Hers D, Luminet O. Absolute and relative stability of alexithymia in alcoholic inpatients undergoing alcohol withdrawal: relationship to depression and anxiety. Psychiatry Res 2008:157:105-13.

[23] Dorard G, Berthoz S, Phan O, Corcos M, Bungener C. Affect dysregulation in cannabis abuser: a study in adolescents and young adults. Eur Child Adolesc Psychiatry 2008;17:274-82.
[24] de Haan H, Joosten E, Wijdeveld T, Boswinkel P, van der Palen J, De Jong C. Alexithymia is not a stable personality trait in patients with substance use disorders. Psychiatry Res 2012;198:123-9.

[25] Marchesi C, Brusamonti E, Maggini C. Are alexithymia, depression, and anxiety distinct constructs in affective disorders? J Psychosom Res 2000;49:43-9.

[26] Hoffart A. State and personality in agoraphobic patients. J Pers Disord 1994;8:333-41.

[27] Lumley MA. Alexithymia and negative emotional conditions. J Psychosom Res 2000;49:51-4.

[28] Mazzi F, Morosini P, de Girolamo G, Lusetti M, Guaraldi GP. Structured clinical interview for DSM-IV axis I disorders. Edizione Italiana. Firenze, Organizzazioni Speciali; 2000.

[29] Hamilton M. The assessment of anxiety states by rating. Br J Med Psychol 1959;32:50-5.

[30] Hamilton M. A rating scale for depression. J Neurol Neurosurg Psychiatry 1960;23:56-62.

[31] Bressi C, Taylor G, Parker J, Bressi S, Brambilla V, Aguglia E, et al. Cross validation of the factor structure of the 20-item Toronto Alexithymia Scale: an Italian multicenter study. J Psychosom Res 1996;41:551-9.

[32] Celikel FC, Kose S, Erkorkmaz U, Sayar K, Cumurcu BE, Cloninger CR. Alexithymia and temperament and character model of personality in patients with major depressive disorder. Compr Psychiatry 2010;51:64-70.

[33] Grabe HJ, Spitzer C, Freyberger HJ. Alexithymia and personality in relation to dimensions of psychopathology. Am J Psychiatry 2004;161:1299-301.

[34] Conrad R, Wegener I, Imbierowicz K, Liedtke R, Geiser F. Alexithymia, temperament and character as predictors of psychopathology in patients with major depression. Psychiatry Res 2009;165:137-44.

[35] Cox BJ, Swinson RP, Shulman ID, Bourdeau D. Alexithymia in panic disorder and social phobia. Compr Psychiatry 1995;36:195-8.

[36] Majohr KL, Leenen K, Grabe HJ, Jenewein J, Nuñez DG, Rufer M. Alexithymia and its relationship to dissociation in patients with panic disorder. J Nerv Ment Dis 2011;199:773-7.

[37] De Berardis D, Serroni N, Campanella D, Carano A, Gambi F, Valchera A, et al. Alexithymia and its relationships with C-reactive protein and serum lipid levels among drug naïve adult outpatients with major depression. Prog Neuropsychopharmacol Biol Psychiatry 2008;32:1982-6.

[38] Luca M, Luca A, Calandra C. Psychomotor retardation and externally oriented thinking in major disorder. Neuropsychiatr Dis Treat 2013;9: $759-66$.

[39] Le HN, Ramos MA, Muñoz RF. The relationship between alexithymia and perinatal depressive symptomatology. J Psychosom Res 2007;62: 215-22.

[40] Marchesi C, Viviani D, Giaracuni G, Paraggio C, De Panfilis C. Premorbid levels of alexithymia in women developing panic disorder. Psychiatry Res 2013 [in press]. http://dx.doi.org/10.1016/j.psychres. 2013.10.030.

[41] Corcos M, Guilbaud O, Speranza M, Paterniti S, Loas G, Stephan P, et al. Alexithymia and depression in eating disorders. Psychiatry Res 2000;9:263-6.

[42] Hintikka J, Honkalampi K, Lehtonen J, Viinamäki H. Are alexithymia and depression distinct or overlapping constructs? A study in a general population. Compr Psychiatry 2001;42:234-9.

[43] Ozsahin A, Uzun O, Cansever A, Guicat Z. The effect of alexithymic features on response to antidepressant medication in patients with major depression. Depress Anxiety 2003;18:62-6.

[44] Costa A, Peppe A, Cantesimo GA, Pasqualetti P, Caltagirone C. Alexithymia in Parkinson's disease is related to severity of depressive symptoms. Eur J Neurol 2006;13:836-41.

[45] Montebarocci O, Codispoti M, Surcinelli P, Franzoni E, Baldaro B, Rossi N. Alexithymia in female patients with eating disorders. Eat Weight Disord 2006;11:14-21.

[46] Haviland MG, Hendryx M, Shaw DG, Henry JP. Alexithymia in women and men hospitalized for psychoactive substance dependence. Compr Psychiatry 1994;35:124-8. 
[47] Uzun AZ, Ates A, Cansever A, Ozsahin A. Alexithymia in male alcoholics: study in a Turkish sample. Compr Psychiatry 2003;44: 349-52.

[48] Farges F, Corcos M, Speranza M, Loas G, Perez-Diaz F, Venisse JL, et al. Alexithymia, depression and drug addiction. Encéphale 2004;30:201-11.

[49] Suslow T, Kersting A, Ohrmann P, Arolt V. A critique of the construct "alexithymia" and its measurement-the weakness of self-report and the opportunities of an objective assessment approach. Z Psychosom Med Psychother 2001;47:153-66.
[50] Kooiman CG, Spinhoven P, Trijsburg RW. The assessment of alexithymia: a critical review of the literature and a psychometric study of the Toronto Alexithymia Scale-20. J Psychosom Res 2002;53: 1083-90.

[51] Vanheule S, Desmet M, Paul Verhaeghe P, Bogaerts S. Alexithymic depression: evidence for a depression subtype? Psychother Psychosom 2007;76:315-6.

[52] Leweke F, Leichsenring F, Kruse J, Hermes S. Is alexithymia associated with specific mental disorders? Psychopathology 2012;45:22-8. 